INPLASY

PROTOCOL

To cite: Hu et al. Clinical Yiguan decoction for liver cirrhosis: protocol of a systematic review and metaanalyses. Inplasy protocol 2020120114. doi:

10.37766/inplasy2020.12.0114

Received: 23 December 2020

Published: 23 December 2020

Corresponding author:

Xingyao Hu

Huxingyao2019@126.com

Author Affiliation: Jiangxi University of Traditional Chinese Medicine

Support: 20202BABL206149.

Review Stage at time of this submission: The review has not yet started.

Conflicts of interest:

None.

\section{Clinical Yi-guan decoction for liver cirrhosis: protocol of a systematic review and meta-analyses}

$\mathrm{Hu}, \mathrm{X}^{1}$; Lu, X2; Guangbin, S³.

Review question / Objective: This study will explore Yi-guan decoction can will be used as one of the non drug therapies to prevent or treat liver cirrhosis.

Condition being studied: At present, Liver Cirrhosis is common in most later liver and gallbladder diseases that its morbidity and mortality seriously affect human health. The limitation and effectiveness of western medicine on liver cirrhosis have become a huge clinical challenge. However, a large number of clinical studies have shown that Yi-guan decoction has become a complementary treatment for liver cirrhosis. Therefore, this systematic review will aim to explore the safety and feasibility of Yi-guan decoction in the treatment of liver cirrhosis.

INPLASY registration number: This protocol was registered with the International Platform of Registered Systematic Review and Meta-Analysis Protocols (INPLASY) on 23 December 2020 and was last updated on 23 December 2020 (registration number INPLASY2020120114).

\section{INTRODUCTION}

Review question / Objective: This study will explore Yi-guan decoction can will be used as one of the non drug therapies to prevent or treat liver cirrhosis.

Condition being studied: At present, Liver Cirrhosis is common in most later liver and gallbladder diseases that its morbidity and mortality seriously affect human health. The limitation and effectiveness of western medicine on liver cirrhosis have become a huge clinical challenge. However, a large number of clinical studies have shown that Yi-guan decoction has become a complementary treatment for liver 
cirrhosis. Therefore, this systematic review will aim to explore the safety and feasibility of Yi-guan decoction in the treatment of liver cirrhosis.

\section{METHODS}

Participant or population: The inclusion of this literature must be a randomized controlled trial, and patients who meet the diagnostic criteria for liver cirrhosis [2] will be included. This article doesn't limit the age, gender and source of the patient that Exclude with other diseases (hypertension, diabetes) in patients with liver cirrhosis.

Intervention: Patients with liver cirrhosis in the Test group must take Yi-guan Decoction as the main treatment (could be combined with other treatment methods or used alone), the control group cann't use Yiguan Decoction.

Comparator: In the control group, intervention means can include blank control, drug (Traditional Chinese medicine

(TCM), western medicine) treatment, routine symptomatic treatment, etc.

Study designs to be included: Primary outcomes. Liver function indicators: Total bilirubin(TBIL), Alanine transaminase(ALT), Aspartate aminotransferase(AST), etc. Secondary outcome indicators (1) total effective rate (2) Nutrition index (3) Survival analysis (4) Adverse events.

Eligibility criteria: The inclusion of this literature must be a randomized controlled trial, and patients who meet the diagnostic criteria for liver cirrhosis [2] will be included.

Information sources: We will conduct a comprehensive literature search in Medline, PubMed, Cochrane Database of Systematic Reviews(Cochrane Library), Embase, Chinese Biomedical Literatures Database(CBM), China National Knowledge Infrastructure(CNKI), Wang Fang Database (WF), Chinese Scientific Journal Database (VIP) from inception to December 2020 without any language restriction. The main subject terms searched:"Yi-guan decoction" "cirrhosis" "liver cirrhosis". The retrieval strategy of Pubmed database is shown in Table 1. Other database retrieval methods will be adjusted in line with database differences.

Main outcome(s): Liver function indicators: Totalbilirubin (TBIL), Alanine transaminase (ALT), Aspartate aminotransferase (AST), etc.

Quality assessment / Risk of bias analysis: If the number of included documents is large (>10), the software Review Manager 5.4 line inverted funnel graph can be used to analyze the bias situation that the shape of the graph can provide reference.

Strategy of data synthesis: Data can be divided into counting data and measurement data. Counting data is expressed by odds ratio (OR) and $95 \%$ confidence interval (Cl); Weighted Mean Difference (WMD) and 95\% confidence interval (CI) is used to represent measurement data. Standardized Mean Difference (SMD) is used when the units are not uniform.

Subgroup analysis: If there will be great heterogeneity in the analysis process, subgroup analysis of the included articles will be performed according to different control measures.

Sensibility analysis: The purpose of sensitivity analysis is going to evaluate the authenticity of meta-analysis. We will use software Stata $\mathbf{1 4 . 0}$ to perform sensitivity analysis.

Country(ies) involved: China.

Keywords: Yi-guan decoction, liver cirrhosis, protocol, systematic review and meta-analysis.

Contributions of each author:

Author 1 - Xingyao Hu.

Author 2 - Xiaonan Lu.

Author 3 - Shang Guangbin. 